\title{
EVALUATING SMARTPHONES COLOR FIDELITY AND METRIC ACCURACY FOR THE 3D DOCUMENTATION OF SMALL ARTIFACTS
}

\author{
M. Gaiani ${ }^{\text {a }}$, F. I. Apollonio ${ }^{\text {a }}$, F. Fantini ${ }^{\text {a, }}$, \\ ${ }^{a}$ Dept. of Architecture - Alma Mater Studiorum University of Bologna, Italy - (marco.gaiani, fabrizio.apollonio, \\ filippo.fantini2)@unibo.it
}

KEY WORDS: Smartphone sensors, Automatic photogrammetry, Participatory sensing, Museums, Smartphone, Color capture \& visualization, 3D modeling

\begin{abstract}
:
Smartphone camera technology has made significant improvements of sensors quality and software camera performance in recent years. Devices as Apple iPhone X and the Samsung Galaxy S9 Plus, allow to reach levels of image resolution, sharpness and color accuracy very close to prosumer SLR cameras, enabling also on-the-fly processing and procedures which were considered impossible to achieve until a few years ago. Starting from these premises, a series of issues and opportunities concerning smartphone application to artifacts documentation will be discussed. In particular, consistency and reliability of both shape and color representation achievable for small-medium artifacts belonging to exhibitions and museum collections. A low-cost, easy-to-use workflow based on low-cost widespread devices will be compared to consolidated digitization pipelines. The contribution focus is based on color accuracy of textured models achievable through smartphones by means of an internally developed application for the achievement of highly reliable developments of raw formats (.DNG) from Apple iPhone X. Color consistency will be calculated in terms of the mean camera chroma relative to the mean ideal chroma in the CIE color metric $\left(\Delta \mathrm{E}^{*}{ }_{00}\right)$ as defined in 2000 by the CIE on the CIEXYZ chromaticity diagram.
\end{abstract}

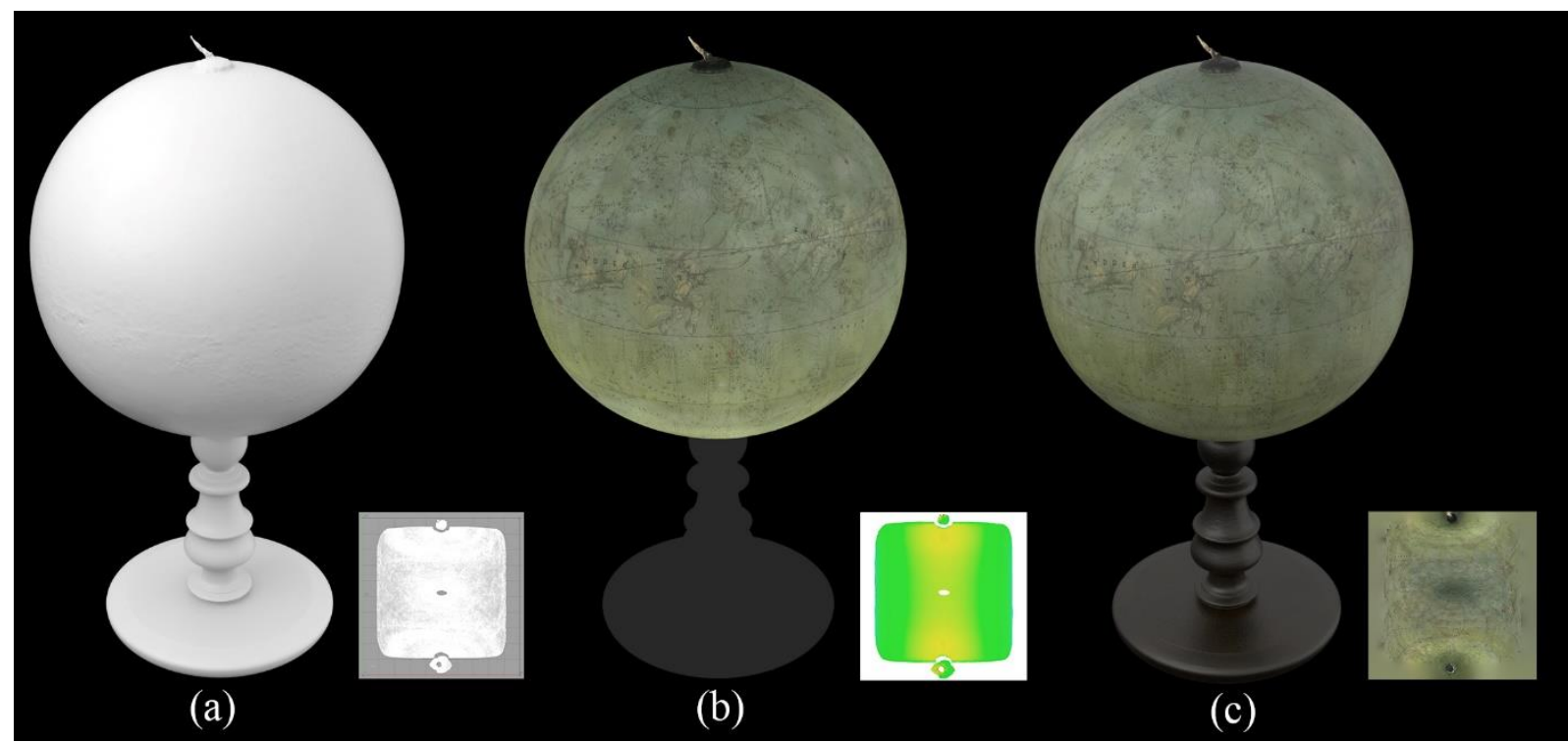

Figure 1. Expected result: (a) reliable model and consistent uv layout; (b) Albedo colour fidelity and controlled distortion of parameterization; (c) Accurate shader definition of the final model.

\footnotetext{
* Corresponding author
} 


\section{INTRODUCTION}

In recent years, smartphone camera technology has made formidable improvements regarding sensors quality and software camera performance, thanks to the flourishing of scientific research in the area. Recent smartphones, such as the Apple iPhone $X$ and the Samsung Galaxy S9 Plus, allow to reach levels of image resolution, sharpness and color accuracy very close to prosumer SLR cameras (DXO, 2019), enabling also on-the-fly processing and procedures which were considered impossible to realize until a few years ago. Furthermore, the current fast integrated LTE communication technology implemented in smartphone devices allows to easily and quickly transfer the recorded pixels to on-board or cloudbased servers for further processing and 3D reconstruction purposes (Kolev et al., 2014; Muratov et al., 2016; Nocerino et al., 2017; TRNIO, 2019). These image-based processes allow to achieve high quality 3D models. Moreover, mobile phones implement extra sensors - gyroscope and accelerometer- that in a not too distant future may open additional possibilities to 3D reconstructions (Kos et al., 2016).

These distinctive features enable devices to have a prominent role in case of massive and low-cost documentation of Cultural Heritage $(\mathrm{CH})$ artefacts. An easy-to-use workflow based on low-cost widespread devices appear as a potentially outstanding solution for the everyday needs of non-expert operators for study, classification, preservation, communication, restoration, etc.

In this paper, we present a semi-automated, easy to use workflow based on a recent smartphone, the Apple iPhone $\mathrm{X}$ (Apple, 2019), and exploiting software solution commercial but low-cost and/or developed by our group for SLR cameras in previous experiences The aim of the solution is to enable a 3D digital culture in the small and medium museums, where most of the heritage objects are preserved. These cases present the severe problem of technological innovation, with special regards to small and medium objects that are also a major asset. We evaluated our solution on the following topics: its capability to (i) provide accurate $3 \mathrm{D}$ geometries and (ii) preserve color fidelity in the final textured 3D models (Figure 1).

This could clarify if the process output is exclusively suitable for communication uses or able to cover all the needs cited above.

\section{AIMS OF THE STUDY}

According to Italian Statistical Institute (ISTAT), Italy has 4,976 public and private museums open to the public, but structures able to attract more than 500 thousand visitors represent less than $1 \%$ of the total (Bertollini et al. 2018). Most museums are characterized by low budgets for preservation, documentation and dissemination of collections, consisting, usually, of micro-/meso-scale objects (Pintus et al., 2016).

In order to set up a procedure capable to give a reliable and consistent solution to micro-/meso-scale object acquisition, three heterogeneous objects were chosen from the Sistema Museale di Ateneo (SMA) of the University of Bologna hosted inside Palazzo Poggi, the headquarters of the University, a remarkable XVI century building (Ottani Cavina, 1988). The acquired datasets correspond to three artefacts representative of the collection and appropriate for the test:

- A statue representing Heracles (Figure 2a): bounding box equal to $100 \times 90 \times 275 \mathrm{~cm}$, made in sandstone, located in the corner of the entrance of the glorious "Aula Carducci";

- A porcupine fish (Diodon antennatus) preserved through a complete taxidermy treatment (Figure 2b): bounding box equal to $35 \times 19 \times 25 \mathrm{~cm}$, with a highly specular skin and tiny details;

- A globe by astronomer Horn d'Arturo (Figure 2c): bounding box equal to $31 \times 31 \times 46 \mathrm{~cm}$, highly reflective regular surface with a considerable Fresnel effect.

These objects were selected with the aim to cover a wide range of types of objects: specific and distinctive shape features, different surveying logistics (affecting SfM network design/planning), different BRDF behavior (from approximated Lambertian surface to highly specular dielectric).

In particular, the porcupine fish and the globe could be acquired under controlled light condition, the Heracles just under semidiffuse light condition.

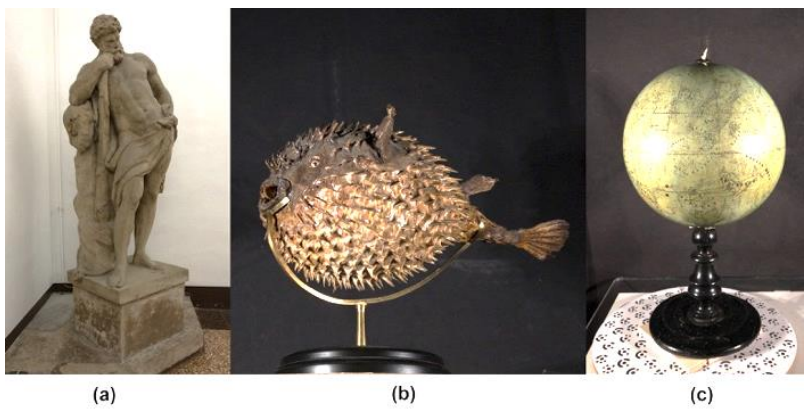

Figure 2. Case studies: (a) Heracles statue; (b) a porcupine fish; (c) Horn d'Arturo's globe.

The aim of the research is to evaluate a low-cost, easy portable and consistent workflow using the following devices and applications (Figure 3):

- Apple iPhone X;

- X-Rite ColorChecker Passport (CC) physical reference chart;

- Array of Relio ${ }^{2}$ spotlight illumination set (www.relio.it): a certified white light source enabling faithful color representation of objects and surfaces illuminated with it;

- An automatic image pre-processing phase carried out by SAT $\&$ HUE Adaptive Fine Tuning (SHAFT) application aimed at the image enhancement: (Gaiani and Ballabeni, 2018);

- A semi-automated photogrammetric pipeline based on Agisoft Metashape (Agisoft, 2019).

The ultimate goal of this evaluation is to verify if our designed and implemented hardware/software workflow is able to return 3D models reliable both from a metric and a radiometric point of view by using smartphone.

\section{RELATED WORKS}

Most of other experiments carried out on 3D models from smartphones are focused on shape/geometry (Nocerino et al., 2017, Poiesi et al., 2017, Garro et al., 2016, Ondruska et al., 2015); no experiments in the field of color corrected reproduction have been carried out on smartphones. Therefore, this is a new research field where the starting point is to apply a method with solid foundations in the field of the colorimetry, in the detail of the faithful color reproduction, and more in general of the image quality in addition to the development and use of dedicated software, to support the well-established automatic photogrammetric pipeline.

About image quality, the IEEE Standard Association define the fundamental test methods for measuring camera phone image quality (for both video and still image) by means of the existing metrics and other information relating to these attributes. The aim is fostering standards-based communication and comparison among manufacturers, and component vendors regarding camera phone image quality. In detail, the IEEE P1858 CPIQ (Camera Phone Image Quality) Standard well frame factors characterizing image quality of smartphone 
cameras behind number of megapixels and potentially source of problems in the photogrammetric process (IEEE CPIQ, 2017). Seven metrics, all based on objective measurements, were used to create the CPIQ standard:

- The metrics include spatial frequency response (SFR);

- Lateral chromatic displacement (LCD);

- Chroma level (CL);

- Color uniformity (CU);

- Local geometric distortion (LGD);

- Visual noise $(\mathrm{VN})$;

- Texture blur (TB) (Baxter et al., 2012; Jin et al., 2017).

The term Texture defines structures that are often randomly oriented and of low contrast. Noise reduction algorithms affect these structures because they cannot be differentiated from noise. This image quality aspect highly depends on the illumination level and is essential for the quality of the image.
In our case $\mathrm{CL}$ and $\mathrm{TB}$ are main factors potentially affecting final results.

The problem of color fidelity of a camera image could be defined as the ability to reproduce colors as they exist in the original scene and could be depicted as the problem of faithfully determining the color and tone level and can be solved by the chromatic and tonal definition (Reinhard et al., 2008).

The fidelity of color reproduction depends on a number of variables, such as the lighting level during the acquisition step, the technical characteristics of the acquisition system (i.e. CMOS sensor of digital camera, lens system, etc.), and the mathematical representation of color information throughout the acquisition and reproduction pipeline. To evaluate color fidelity, the CIEDE colorimetry standards of the CIE organization have been acknowledged the most usable color difference metrics, approved as a joint international standard between the ISO and CIE organizations (ISO/CIE 11664-6, 2014; Habekost, 2013).

$\begin{array}{cc}\text { TOOLS } & \text { IMAGE } \\ & \text { PRE- PROCESSING } \\ \text { APPLE IPHONE X } & \text { TARGET } \\ \text { X-RITE MSCCC COLOR- } & \text { LOCALIZATION } \\ \text { CHECKER CLASSIC } & \text { NOISE REDUCTION } \\ \text { RELIO2 ILLUMINATION } & \text { INITIAL WHITE } \\ \text { SET } & \text { BALANCE } \\ \text { ROTARY TURNTABLE } & \triangle \mathrm{SE}_{\text {wo EVALUATION }} \\ \text { WITH RAD CODED } & \text { SHAFT SCRIPT } \\ \text { TARGET } & \text { COLOR BALANCED } \\ \text { BLACKOUT CURTAIN } & \text { OUTPUT } \\ \text { SET } & \end{array}$

In particular, we used the last version is the CIEDE2000 (Sharma et al., 2005) that compensates better for perceptual non-uniformities of the $\mathrm{L}^{*} \mathrm{a} * \mathrm{~b} *$ color space and thus correlates better with perceptual color difference than earlier equations (Mokrzycki and Tatol, 2011):

$\Delta E_{00}^{*}=\sqrt{\left(\frac{\Delta L^{*}}{k_{L} S_{L}}\right)^{2}+\left(\frac{\Delta C^{*} a b}{k_{c} S_{c}}\right)^{2}+\left(\frac{\Delta H^{*} a b}{k_{H} S_{L H}}\right)^{2}+R_{T} \frac{\Delta C^{\prime}}{k_{c} S_{c}} \frac{\Delta H^{\prime}}{k_{H} S_{c H}}}$

The equation represents, in practice, the Euclidean distance in $\mathrm{L} * \mathrm{a} * \mathrm{~b} *$ color space between captured image values and measured values. Normally the $\mathrm{L}^{*} \mathrm{a} \mathrm{b}^{*}$ values are average values of uniformly colored test patches as the CC color chart, which color values are known precisely.

In the context of the color fidelity, evaluation exposure plays an important role, mainly the small sensors of the smartphone cameras. Generally, exposure can be adjusted by using the three variables aperture, exposure time and amplification (exposure index). The maximum aperture is limited by size and lens design. The maximum exposure time is limited by handshake if there is not image stabilization built in and the maximum amplification is limited by noise performance. Exposure is a crucial problem in the tonal rendering that is scene dependent, and a too dark or a too clear image could avoid a reliable color reproduction.

Color fidelity in the field of 3D models construction from a photogrammetric process, basically consists in ensuring a faithful reproduction of the captured images to be used in the photogrammetric pipeline, and the resulting texture mapping from the process. In this paper, we focus on the first problem, that is sensor-dependent, while the second could be placed in a class of problem more linked to the photogrammetric solution.

\section{DEVICES TECHNICAL}

\subsection{Phone and SLR cameras}

The Apple iPhone $\mathrm{X}$ presents levels of image resolution, sharpness and color accuracy very close to prosumer SLR cameras. It presents a dual-12MP (wide+telephoto) camera setup. The wide-angle sensor sits behind a $4 \mathrm{~mm}$ and f/1.8 lens; the telephoto presents a $6 \mathrm{~mm}$ and $\mathrm{f} / 2.4$ lens. The iPhone $\mathrm{X}$ has Sony Exmor RS sensors, the wide-angle camera has a $1.22 \mu \mathrm{m}$ pixel size, and the telephoto camera has a $1.0 \mu \mathrm{m}$ pitch as before. It is supported by ad hoc developed image processor and noise reduction algorithm. Camera sensor is manufactured using deep trench isolation tech, a technology also available on Sony Xperia (DTI) and Samsung Galaxy (ISOCELL) smartphones for better pixel isolation on the sensor itself, which prevents light leakage between the neighboring pixels and thus improves the overall photo quality. iPhone $\mathrm{X}$ relies on a 6P (six-lens plastic lens array) lens design. Optical image stabilization is available for both wide angle and telephoto cameras, and could be disabled using Pro capture software as Adobe Lightroom CC.

The chosen reference digital SLR is a Nikon 5200 camera, with a $23.5 \times 15.6 \mathrm{~mm}$ APS-C CMOS sensor, and an image resolution of $6000 \times 4000 \mathrm{px}$, coupled with a zoom lens 18-55 $\mathrm{mm}$.

\subsection{Illumination setup}

A key role of the experiments, in acquisition of medium-small objects, is played by Relio ${ }^{2}$ illumination devices adopted during the image capture (Figure 4). It is a very small illuminator $(35 \times 35 \times 35 \mathrm{~mm}, 80 \mathrm{~g})$ emitting continuous spectrum light at a $\mathrm{CCT}$ of $4000^{\circ} \mathrm{K}$, a neutral white with high color rendering, has a brightness of 40000 lux at $0.25 \mathrm{~m}$, and has a CRI> $95 \%$.

Each Relio ${ }^{2}$ is individually calibrated and the light emitted is measured using a spectrophotometer allowing to calculate the white balance mathematically - and not empirically. 
This light source avoids the excessive emission of color, the emission of harmful UV and IR radiation and a typical LED problem, that is the flicker effect due to the harmonic ripple that generate the traditional electronics for driving the LEDs in current control and those for adjusting the brightness. The illuminator therefore retains a series of basic characteristics that make it extremely suitable for achieving a proper textured model.

\subsection{Acquisition setup}

The Heracles statue dataset (378 images acquired with iPhoneX, 316 with Nikon D5200) consists of convergent images and some vertical camera rolls taken all around the statue.

The statue features some emblematic situations, i.e., 3D scenes (non-coplanar), textureless surfaces (due to sandstone) and illumination changes (light and shadow). The location is illuminated by indirect fluorescent light, coming from above.

For the porcupine fish and Horn d'Arturo's globe the illumination and photographic setup consists of a black canvas background, a revolving graduated object holder base, and two $2 \times 2$ arrays of Relio ${ }^{2}$ spotlights placed about $45^{\circ}$ to the lens axis, illuminating each side of the artwork.

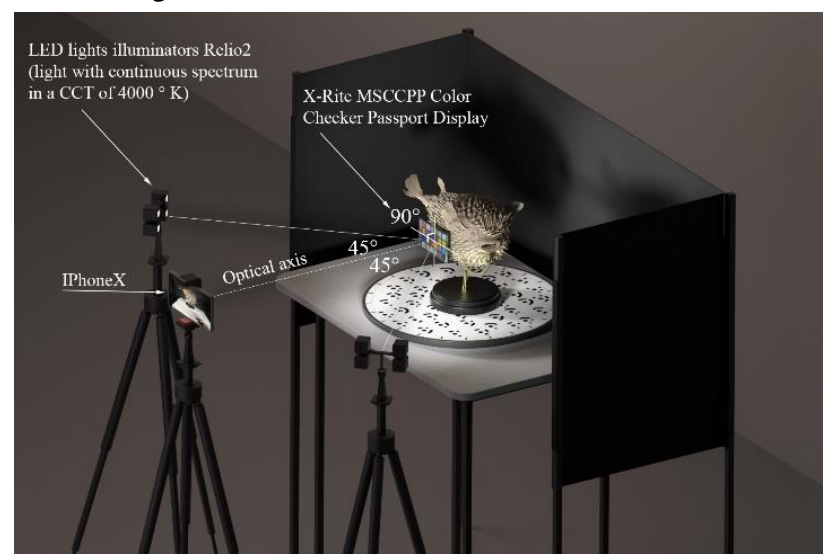

Figure 4. Illumination setup of an artwork with Relio ${ }^{2}$.

These two datasets consist of four groups of 18 convergent images, taken all around the artwork at four different heights (Figure 4).

\section{METHODOLOGY}

In our tests we employed a well-established 3D reconstruction pipeline, successfully tested in many heritage case studies (Remondino et al., 2016; Apollonio et al., 2017a; Apollonio et al., 2017b) starting from RAW photographs and consisting of a customized, optimized and automated procedure, able to improve the traditional solution based on SfM and dense stereo matching.

The first step deals with a completely automated image preprocessing, aimed at enhancing photograph quality (radiometric calibrated images) carried out by SHAFT application (SAT \& HUE Adaptive Fine Tuning (Gaiani and Ballabeni, 2018), and yielding better results during the following photogrammetric processing, i.e. image orientation and dense image matching (Gaiani et al. 2016; Gaiani et al., 2017).

The second step consist in a photogrammetric-based 3D model construction established on the automated pre-processing procedure illustrated in (Apollonio et al., 2014), allowing a robust image matching starting from RAW photographs. The remaining phases of the automatic photogrammetric pipeline (Structure-from-Motion, Bundle Adjustment, dense stereo matching), the mesh reconstruction and the texture mapping are based on the automation and customization of Metashape, a state-of-the-art commercial stand-alone software product, finalized to generate 3D spatial data (Agisoft Metashape, 2019). The color analysis is based on a CC target and it includes: a physical reference chart acquired under the current illumination (corresponding to the illumination to be discarded); a color space reference chart with the ideal data values for the chart; a way to relate or convert the device color space to the reference color space chart; and a way to measure and show errors in the device's rendering of the reference chart. Color accuracy was computed in terms of the mean camera chroma relative to the mean ideal chroma in the CIE color metric $\left(\Delta E^{*}{ }_{00}\right)$ as defined in CIEDE2000. The $\Delta E^{*}{ }_{00}$ calculation was made using the Imatest Master software version 4.5 (Imatest, 2018).

The smartphone-based results will be compared against ground truth reference results concerning both shape and color reliability. The accuracy evaluation of the mesh results is done using two active sensors: a phase-shift Faro Focus X 130 (for meso-scale objects artefacts, i.e. Heracles's statue), and a popular and widespread ranging device based on multiple fringe projection NextEngine Desktop laser scanner (nominal 3D point accuracy up to $0.127 \mathrm{~mm}$ ).

\subsection{Radiometric processing pipeline}

Our color processing essentially consists in a thoughtful revision of a classic pipeline of image radiometric calibration and enhancement to achieve color corrected images and in an adaptation to the specific camera phone features and problems. We adopted a technique that uses a set of differently colored samples measured with a spectrophotometer, taking as target the popular and consistent CC.

CC combines three photographic targets into one pocket size protective, multi-positionable case (Myers, 2010). The CC consists of 24 painted squares with known reflectance applied to paper, ranging from uniform gray lightness scale to six primary colors, and to approximations of medium light and medium dark human skin, blue sky, the front of a typical leaf, and blue chicory flower.

The steps are executed automatically with an application consisting in a RAW image processing implemented in MATLAB and supported by DCRaw, allowing image demosaicing, white balance, output file in a rendered color space, gamma correction, brightness control, 8-bit/16-bit conversion. Our automatic workflow is described in Gaiani et al. (2017). The so-called new SHAFT (is inspired by the Adobe Camera Raw (ACR) calibration scripts coming from Bruce Fraser's calibration procedure for successive approximations (RAGS, 2018) and is coupled with a polynomial regression, introducing several optimizations and enhancement to the original algorithm.

The developed solution differs from the original technique for the number and types of tests done along the processing and for the algorithm used to find the best variation from the original values of the selected parameters (exposure, contrast, white balance, hue and saturation on each RGB channel).

In the color correction process, a key-point is the use of appropriate color spaces in which to apply the color correction algorithms given above and the scene-referred non-linear color space (Süsstrunk, et al., 1999). The processing color space used is the CIEXYZ taking in account the consideration that using this color space, errors calculated by the least squares fitting algorithms allows to produce final corrected images that look more closely matched to the original image (Lukac and Plataniotis, 2007). Our final color space is the rendered space sRGB, the current IEC 61966-2-1 default color space for multimedia application. 
We move then in the well-delineated context in which (Stamatopoulos, et al., 2012) demonstrated that a pre-processing approach for RAW imagery can yield significant photogrammetric accuracy improvements over those obtained with JPEG. When you face RAW from SLR traditional digital camera only the basic in-camera processing could be retained.

However, this is not fully possible for smartphone where images appearance is strictly linked with the in-camera image processing. iPhoneX uses as RAW file format the Adobe Digital Negative (DNG), that is both a RAW image format and a format that supports "non-RAW", or partly processed images. The iPhone X record photographs in the Apple Display P3 color space, a variant of the DCI-P3 color space, using the illuminant D65 instead of the D50. DCI-P3 is 25\% larger than the sRGB color space that most devices use, most web browsers assume and also is supported by the OpenGL API libraries, and defined at 16 bits/channel color depth.

To minimize these unwanted modifications, we customized the processing software to redo the original state of the file basically about two major problems affecting color fidelity: color space and Gamma correction. At the end of the processing to ensure that the images we used display properly on a wide variety of browsers and devices, we converted the originals from DCI-P3 to sRGB. This can slightly reduce the richness of color in some cases from what one would see when viewing the original images on a DCI-P3-calibrated display with appropriate software.

Another improvement of the first version of the SHAFT software consists in the integration in the demosaicing and color correction process of a denoise processing to overcome the high noise typical of the small sensor and lens size of modern mobile phone cameras in the low light imaging situations (i.e. the standard condition of the museum objects).

Image noise is defined in the ISO 15739 standard as "unwanted variations in the response of an imaging system" (ISO, 2013). In digital cameras noise consists of two parts: fixed noise (sensor dark current noise and thermal (resistive) noise) and shot (photon) noise, which increases with the square root of the mean number of photons striking the pixels. It has to be noted that the total noise value is a complex mixture of several noise sources of lens system, pixel size, sensor technology and manufacturing, image processing pipeline, ISO speed, exposure time, RAW conversion. Noise scales strongly with pixel size, becoming ugly in compact digital cameras and camera phones with tiny sensors, especially at high ISO speeds or in dim light, and affected by sensor technology and manufacturing quality (Peltoketo, 2015).

The noise amount can be partially controlled by camera parameters, like ISO speed and exposure time, and denoising algorithms. However, the separation of different noise sources from the total noise value is a difficult task and the denoise processing need to be used carefully.

To avoid information loss, a two-step process is necessary:

Noise measurement;

Denoise based on previous measurement.

Noise measurements should ideally correlate with perceived appearance, referenced to the original scene not affected by the tonal response (gamma) of the camera or the RAW converter, and simple enough to interpret without difficulty.

Noise measurements usually refer to Root Mean Square noise $(R M S)$, which is same of the Standard deviation of the signal $S$ :

$$
\text { RMS Noise }=\mathrm{N}=\sigma(\mathrm{S})
$$

where $\sigma$ denotes the standard deviation and $S$ can be the signal. Signal-to-Noise Ratio (SNR) is a measurement derived from noise that is often considered more important than noise itself. It is expressed as simple ratio:

$$
S N R=S / N
$$

Nowadays, following the ISO 15739, the noise measurements of the digital cameras are mainly based on OECF tests chart. The objective noise measurement is based on noise and signal to noise (SNR) calculations of uniform gray patches of a test chart. When the reference target is uniformly gray and correctly illuminated, all variations in the captured image can be judged as noise.

The developed noise measurement is instead based on the RMS noise for the 24 patches of the CC measured in the XYZ linear color space and the normalized to $1-255$ in the RGB color space. This solution is not very accurate but correctly enable our denoiser, allowing to accurately identify the algorithm parameters to be done as input.

Using measured noise level, a denoise strategy was applied with the main goals proper of the automatic photogrammetry field:

- perceptually flat regions should be as smooth as possible, and noise should be completely removed from these regions;

- image boundaries should be well preserved and not blurred;

- texture detail should not be lost;

- the global contrast should be preserved (i.e., the lowfrequencies of denoised and input images should be equal); - no artifacts should appear in the denoised image.

These allow to minimize remove signal, blob shape and intensity areas distortion to have efficient keypoint extraction and image matching processing, reliable color reproduction.

To achieve these goals in a previous experience (Gaiani et al., 2016), we developed an in-house method (named CBM3D-new) starting from the Color Block Matching 3D (CBM3D) approach (Dabov et al., 2007a), a color variant of Block Matching 3D (BM3D) filter (Dabov et al., 2007b). This denoiser is basically a learned approach patch-based method exploiting both local and non-local redundancies and "self-similarities" in the images.

In detail, we evaluated the parameter $\sigma$ (the standard deviation of zero mean additive white Gaussian noise) measured in an 8bit scale as in the original code, that, as noted also by Lebrun (2012), is a very sensitive parameter of the algorithm. Using different values of $\sigma$, the algorithm switches between the five profiles corresponding to different parameters in the algorithm: "fast" - Fast Profile (default); "Ic" - Low Complexity Profile; "np" - Normal Profile; "high" - High Profile; "vn" - Very Noisy Profile.

In practice, we skipped the "lc" and the "high" profiles, mainly differentiating the three cases of $\sigma<10,40>\sigma>10, \sigma>40$. For the first case we used the original parameters, for the last two cases we used the parameters suggested by Lebrun (2012), consisting basically in different values for the maximum thresholds for the distance between two similar patches.

\section{OUTCOMES}

To report the workflow performances, the accuracy evaluation of the polygonal model reconstruction results was done using Terrestrial Laser Scanning (Faro Focus X 130 and NextEngine) data as reference, and then the two image-based models compared to one another. The same Ground Sampling Distance is used (a grid of around $2 \times 2 \mathrm{~mm}$ ).

Figures 3-6 and the related Tables 7-10 show the results of model comparison: mesh deviation distribution in false color; histogram of point distribution errors; average; Standard Deviation and $1 \sigma$.

All the results show an excellent reliability and consistency, for Heracles model, with a Standard Deviation ranging from 1,87 to $1,93 \mathrm{~mm}$, and an Average distance from 1,45 to $1,56 \mathrm{~mm}$; for Globe model, with a Standard Deviation ranging from 1,24 to $1,87 \mathrm{~mm}$, and an Average distance from 1,04 to $1,45 \mathrm{~mm}$.

For what concern color accuracy reproduction according to the above-mentioned procedure, the $\Delta \mathrm{E}^{*}{ }_{00}$ of the $\mathrm{CC}$ have been 
evaluated for all the datasets acquired using iPhoneX and Nikon D5200 (Tables 11-16). Each dataset includes 9 CC with different orientation in the space.

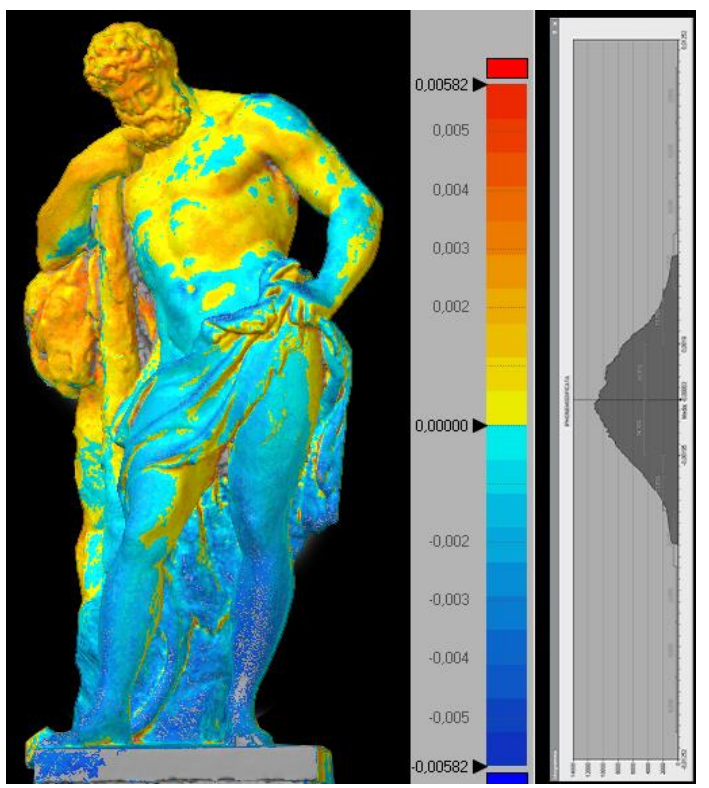

Figure 5. Heracles. iPhoneX based modeling comparison with the ground truth TLS data: mesh deviation distribution in false color; histogram of point distribution errors

\begin{tabular}{|c|c|c|}
\hline \multicolumn{3}{|c|}{ Mesh Deviation Result: Heracles iPhoneX-Faro TLS } \\
\hline Min.: & $-0,005 \mathrm{~m}$ & Int. Sigma \\
\hline Max.: & $0,005 \mathrm{~m}$ & $\begin{array}{l}\text { (Sigma / No. of Poly- } \\
\text { Vertices / \%) }\end{array}$ \\
\hline Media: & $-0,00003 \mathrm{~m}$ & $\pm 1 \sigma / 923190 / 67,41295 \%$ \\
\hline RM & $0,00193 \mathrm{~m}$ & $\pm 2 \sigma / 1301364 / 95,02788 \%$ \\
\hline Standard Dev.: & $0,00193 \mathrm{~m}$ & $\pm 3 \sigma / 1369455 / 100 \%$ \\
\hline Var & $0,00371 \mathrm{~m}$ & $\pm 4 \sigma / 1369455 / 100 \%$ \\
\hline +Avg.: & $0,00156 \mathrm{~m}$ & $\pm 5 \sigma / 1369455 / 100 \%$ \\
\hline \multicolumn{2}{|l|}{ Total: } & $1.552 .852 \mathrm{v}$ \\
\hline \multicolumn{2}{|l|}{ Valid: } & 1.369.455 vertices \\
\hline
\end{tabular}

Table 7. Mesh Deviation Result: Heracles iPhoneX-Faro TLS

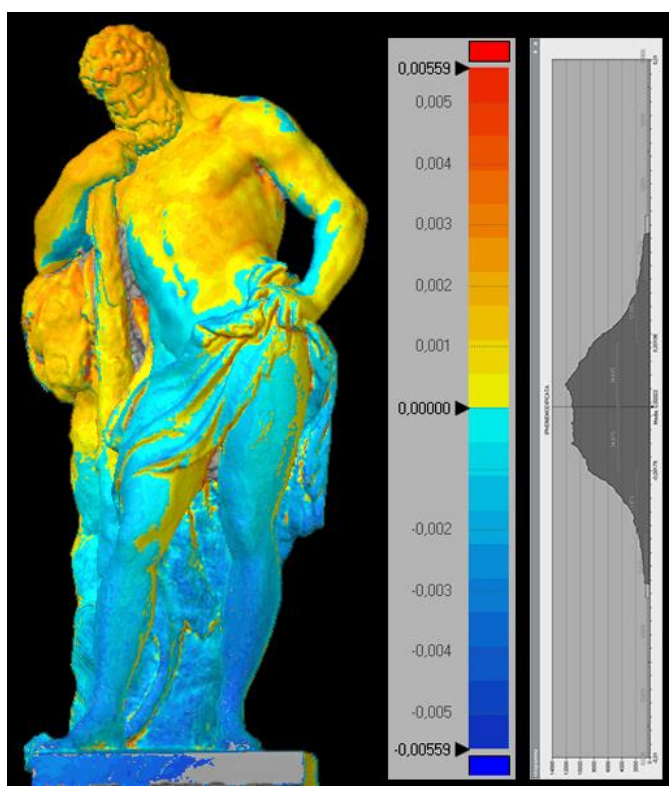

Figure 6. Heracles. iPhoneX and Nikon D5200 based modelling comparison: mesh deviation distribution in false color; histogram of point distribution errors

\begin{tabular}{|c|c|c|}
\hline \multicolumn{3}{|c|}{ Mesh Deviation Result: Heracle iPhoneX-Nikon D5200 } \\
\hline Min: & $-0,005 \mathrm{~m}$ & Int. Sigma \\
\hline Max.: & $0,005 \mathrm{~m}$ & $\begin{array}{l}\text { (Sigma / No. of Poly- } \\
\text { Vertices / \%) }\end{array}$ \\
\hline Media: & $-0,00013 \mathrm{~m}$ & $\pm 1 \sigma / 685734 / 67,93515 \%$ \\
\hline RMS: & $0,00187 \mathrm{~m}$ & $\pm 2 \sigma / 958658 / 94,97352 \%$ \\
\hline Standard Dev.: & $0,00187 \mathrm{~m}$ & $\pm 3 \sigma / 1009395 / 100 \%$ \\
\hline Var.: & $0,00349 \mathrm{~m}$ & $\pm 4 \sigma / 1009395 / 100 \%$ \\
\hline+ Avg. & $0,00145 \mathrm{~m}$ & $\pm 5 \sigma / 1009395 / 100 \%$ \\
\hline \multicolumn{2}{|l|}{ Total: } & 1.293.086 vertices \\
\hline \multicolumn{2}{|l|}{ Valid: } & 1.009.395 vertices \\
\hline
\end{tabular}

Table 8. Heracle iPhoneX-Nikon D5200: Mesh Deviation Result

From the comparison of the values one can see that for Globe and Porcupine case studies (i.e. in the presence of controlled light) the $\Delta \mathrm{E}^{*}{ }_{00}$ mean error values of SLR camera and camera phone are almost identical, while in the case of the Heracles case study the values differ by less than one $\Delta \mathrm{E}^{*}{ }_{00}$ (i.e. the differences in color they are indistinguishable). The denoise does not give benefit on color accuracy but improves the general rendering of the texture, minimizing color fluctuations.

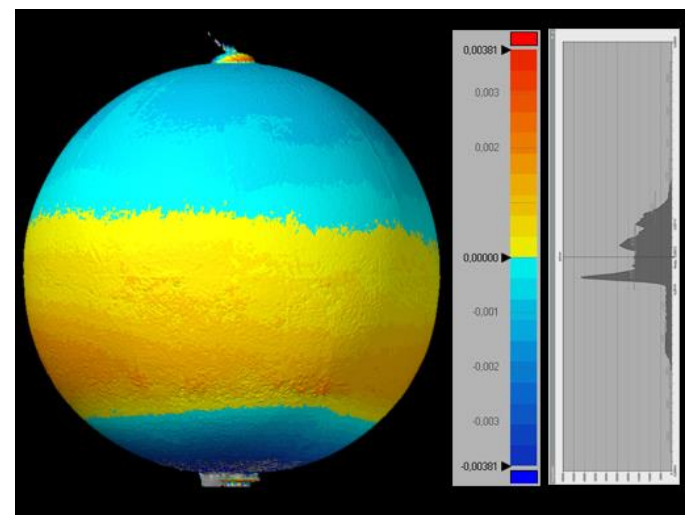

Figure 7. Globe. iPhoneX based modeling comparison with the ground truth NextEngine data: mesh deviation distribution in false color; histogram of point distribution errors

\begin{tabular}{|l|r|r|}
\hline \multicolumn{3}{|l|}{ Globe. Mesh Deviation Result: iPhoneX- NextEngine } \\
\hline Minimo: & $-0,00499 \mathrm{~m}$ & $\begin{array}{l}\text { Int. Sigma } \\
\text { Max.: }\end{array}$ \\
& $0,00496 \mathrm{~m}$ & $\begin{array}{l}\text { (Sigma / No. of Poly- } \\
\text { Vertices / \%) }\end{array}$ \\
Media: & $0,00019 \mathrm{~m}$ & $\pm 1 \sigma / 1269125 / 73,83781 \%$ \\
RMS: & $0,00126 \mathrm{~m}$ & $\pm 2 \sigma / 1612120 / 93,79329 \%$ \\
Standard Dev.: & $0,00124 \mathrm{~m}$ & $\pm 3 \sigma / 1701387 / 98,98685 \%$ \\
Var.: & $0,00154 \mathrm{~m}$ & $\pm 4 \sigma / 1718791 / 99,99942 \%$ \\
+Avg.: & $0,00104 \mathrm{~m}$ & $\pm 5 \sigma / 1718801 / 100 \%$ \\
\hline Total: & 2.642 .952 vertices \\
Valid: & & 1.718 .801 vertices \\
\hline
\end{tabular}

Table 9. Globe. iPhoneX-Faro TLS: Mesh Deviation Result 


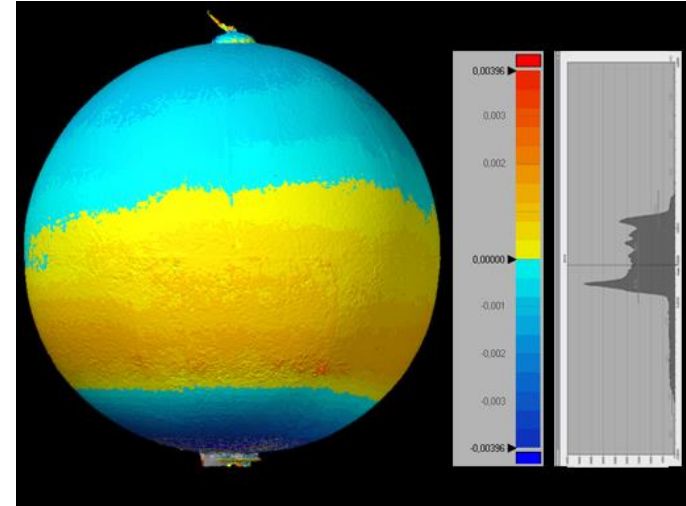

Figure 8. Globe. iPhoneX and Nikon D5200 based modeling comparison: mesh deviation distribution in false color; histogram of point distribution errors

\begin{tabular}{|l|r|r|}
\hline \multicolumn{3}{|l|}{ Globe. Mesh Deviation Result: iPhoneX- Nikon D5200 } \\
\hline Min: & $0,00499 \mathrm{~m}$ & $\begin{array}{l}\text { Int. Sigma } \\
\text { (Sigma / No. of Poly- } \\
\text { Max.: }\end{array}$ \\
& $0,00103 \mathrm{~m}$ & $\begin{array}{r}\text { Vertices / \%) } \\
\pm 1 \sigma / 631160 / 82,82668 \%\end{array}$ \\
Avg: & $0,00136 \mathrm{~m}$ & $\pm 2 \sigma / 710870 / 93,28697 \%$ \\
RMS: & $\pm 3 \sigma / 734855 / 96,4345 \%$ \\
Dev.Standard: & $0,00089 \mathrm{~m}$ & \pm 360 \\
Var.: & $0,0008 \mathrm{~m}$ & $\pm 4 \sigma / 759467 / 99,66432 \%$ \\
+Avg.: & $0,00103 \mathrm{~m}$ & $\pm 5 \sigma / 762025 / 100 \%$ \\
-Mediana: & $0 \mathrm{~m}$ & $\pm 6 \sigma / 762025 / 100 \%$ \\
\hline Total: & & 1.161 .146 vertices \\
Valid: & & 762.025 vertices \\
\hline
\end{tabular}

Table 10. Globe. iPhoneX-Nikon D5200: Mesh Deviation Result

In general, we observe how controlled light conditions lead to relevant benefits (more than $1 \Delta \mathrm{E}^{*}{ }_{00}$ of color difference) especially in the case of the camera phone.

\begin{tabular}{|l|c|c|c|c|}
\hline \multirow{2}{*}{ Mean error } & \multicolumn{2}{|c|}{$\Delta \mathbf{E}^{*}{ }_{00}$ Mean } & \multicolumn{2}{c|}{$\Delta \mathbf{E}^{*}{ }_{00}$ Max } \\
\cline { 2 - 5 } & No Den. & Denoise & No Den. & Denoise \\
\hline St. deviation & $\mathbf{3 , 4 5}$ & $\mathbf{3 , 3 8}$ & $\mathbf{8 , 1 9}$ & $\mathbf{7 , 8 1}$ \\
\hline
\end{tabular}

Table 11. Apple iPhoneX - Heracles statue data set: $\Delta \mathrm{E}^{*}{ }_{00}$

\begin{tabular}{|l|c|c|c|c|}
\hline \multirow{2}{*}{ Mean error } & \multicolumn{2}{|c|}{$\Delta \mathbf{E}^{*}{ }_{00}$ Mean } & \multicolumn{2}{c|}{$\Delta \mathbf{E}^{*}{ }_{00}$ Max } \\
\cline { 2 - 5 } & No Den. & Denoise & No Den. & Denoise \\
\hline St. deviation & $\mathbf{2 , 3 0}$ & $\mathbf{2 , 3 1}$ & $\mathbf{6 , 2 8}$ & $\mathbf{6 , 2 3}$ \\
\hline
\end{tabular}

Table 12. Apple iPhoneX - Horn d'Arturo Globe: $\Delta \mathrm{E}^{*}{ }_{00}$

\begin{tabular}{|l|c|c|c|c|}
\hline \multirow{2}{*}{} & \multicolumn{2}{|c|}{$\Delta \mathbf{E}^{*}{ }_{00}$ Mean } & \multicolumn{2}{c|}{$\Delta \mathbf{E}^{*}{ }_{00}$ Max } \\
\cline { 2 - 5 } & No Den. & Denoise & No Den. & Denoise \\
\hline Mean error & $\mathbf{2 , 1 2}$ & $\mathbf{2 , 1 5}$ & $\mathbf{5 , 2 7}$ & $\mathbf{5 , 2 7}$ \\
\hline St. deviation & $\mathbf{0 , 2 3}$ & $\mathbf{0 , 1 6}$ & $\mathbf{0 , 2 5}$ & $\mathbf{0 , 3 8}$ \\
\hline
\end{tabular}

Table 13. Apple iPhoneX - Porcupine fish: $\Delta \mathrm{E}^{*}{ }_{00}$

\begin{tabular}{|l|c|c|}
\hline & $\Delta \mathbf{E}^{*}{ }_{00} \mathrm{Mean}$ & $\Delta \mathbf{E}^{*}{ }_{00} \mathrm{Max}$ \\
\hline Mean error & $\mathbf{2 , 5 7}$ & $\mathbf{7 , 2 6}$ \\
\hline Standard deviation & $\mathbf{0 , 1 6}$ & $\mathbf{0 , 9 8}$ \\
\hline
\end{tabular}

Table 14. Nikon D5200 - Heracle statue data set: $\Delta \mathrm{E}^{*}{ }_{00}$

\begin{tabular}{|l|c|c|}
\hline & $\Delta \mathbf{E}^{*}{ }_{00} \mathrm{Mean}$ & $\Delta \mathrm{E}^{*}{ }_{00} \mathrm{Max}$ \\
\hline Mean error & $\mathbf{2 , 2 2}$ & $\mathbf{4 , 3 6}$ \\
\hline
\end{tabular}

\begin{tabular}{|c|c|c|}
\hline Standard deviation & $\mathbf{0 , 3 3}$ & 1,86 \\
\hline & $\Delta \mathrm{E}^{*}{ }_{00}$ Mean & $\Delta \mathrm{E}^{*}{ }_{00} \mathrm{Max}$ \\
\hline Mean error & 2,19 & 4,25 \\
\hline \begin{tabular}{|l|} 
Standard deviation \\
\end{tabular} & $\mathbf{0 , 1 2}$ & $\mathbf{0 , 0 7}$ \\
\hline
\end{tabular}

Texture map is finally color corrected in order to overcome a limitation of Metashape, which does not manage correctly the color, producing unwanted artefact and - generally - color differences even considerable due to mismatches between the color spaces (Apollonio et. al., 2017a) (Figure 9).

\section{CONCLUSIONS}

Smartphone camera technology has made significant improvements of sensors quality and software camera performance in recent years, reaching levels of image resolution, sharpness and color accuracy very close to prosumer SLR cameras, enabling also on-the-fly processing and procedures which were considered impossible to realize until few years ago. The achievement of high-quality 3D models with reliable color texture applied using camera smartphones can be considered as a milestone, since it would mark the beginning of a new era in the museum management field, allowing not only the conservators and restorers, but also average users, to replicate accurate low-cost $3 \mathrm{D}$ models in a short time. This would therefore constitute an undeniable opportunity to preserve, document and disseminate contents concerning smallmedium artifacts belonging to exhibitions and museum collections.

We demonstrated that a user-friendly development of a lowcost, easy-to-use workflow, based on low-cost widespread devices is today an achieved milestone.

\section{ACKNOWLEDGEMENTS}

Authors would like to thank Roberto Balzani and Annalisa Managlia of the University of Bologna SMA

\section{REFERENCES}

Agisoft Metashape, 2019. https://www.agisoft.com/ (6 March 2019).

Apollonio, F., Ballabeni, A., Gaiani, M., Remondino, F., 2014. Evaluation of feature-based methods for automated network orientation. Int. Arch. Photogramm. Remote Sens. Spatial Inf. Sci., XL(5), pp. $47-$ 54.

Apple, 2019. www.apple.com/iphone-x (6 March 2019).

Apollonio, F.I., Gaiani, M., Basilissi, W., Rivaroli, L., 2017a. Photogrammetry driven tools to support the restoration of open-air bronze surfaces of sculptures: an integrated solution starting from the experience of the Neptune Fountain in Bologna. Int. Arch. Photogramm. Remote Sens. Spatial Inf. Sci., XLII-2/W3, 47-54. doi.org/10.5194/isprsarchives-XLII-2-W3-47-2017.

Apollonio, F.I., Ballabeni, M., Bertacchi, S., Fallavollita, F., Foschi, R., Gaiani, M., 2017b. From documentation images to restauration support tools: a path following the Neptune Fountain in Bologna design process. Int. Arch. Photogramm. Remote Sens. Spatial Inf. Sci., XLII-5/W1, 329336. doi.org/10.5194/isprs-archives-XLII-5-W1-329-2017.

Baxter, D., Cao, F., Eliasson, H., Phillips, J.B., 2012. Development of the I3A CPIQ spatial metrics. Proc. SPIE, 8293, 829302. doi.org/10.1117/12.905752. 
Bertollini, M., Caramis, A., D’Elia, M., Prisco, M.R., Talice, S., 2018. La valorizzazione del patrimonio museale: i percorsi museali 2015. https://www.slideshare.net/slideistat/ (10 March 2019).

Dabov, K., Foi, A., Katkovnik, V., Egiazarian, K., 2007a. Colour image denoising via sparse 3D collaborative filtering with grouping constraint in luminance-chrominance space. 2007 IEEE ICIP Proceedings, 313316. doi.org/10.1109/icip.2007.4378954.

Dabov, K., Foi, A., Katkovnik, V., Egiazarian, K., 2007b. Image denoising by sparse 3D transform-domain collaborative Filtering. IEEE Trans. Image Process., 16(8), 2080-2095. doi.org/10.1109/TIP.2007.90123

DXO, 2019. www.dxomark.com/ (6March 2019).

Gaiani, M., Remondino, F., Apollonio, F.I., Ballabeni, A., 2016. An Advanced Pre-Processing Pipeline to Improve Automated Photogrammetric Reconstructions of Architectural Scenes. Remote Sensing, 8(3), 178. doi.org/10.3390/rs8030178.

Gaiani, M., Apollonio, F.I., Ballabeni, A., Remondino, F., 2017. Securing colour fidelity in 3D architectural heritage. Sensors, 17(11), 2437. doi.org/10.3390/s17112437.

Gaiani, M., Ballabeni, A., 2018. SHAFT (SAT \& HUE Adaptive Fine Tuning), a new automated solution for target-based colour correction. Colour and Colorimetry. Multidisciplinary Contributions, XIVB, 69-80.

Garro, V., Pintore, G., Ganovelli, F., Gobbetti E., Scopigno, R., 2016. Fast Metric Acquisition with mobile devices. VMV '16 Proceedings. Eurographics Association, Aire-la-Ville, 29-36.
Habekost, M., 2013. Which colour differencing equation should be used?. International Circular of Graphic Education and Research, 6, 20-33.

IEEE P1858, IEEE Standard for Camera Phone Image Quality (CPIQ), May 2017

Imatest, 2018. www.imatest.com (6 March 2019)

ISO 15739: 2013. Photography-Electronic Still-Picture Imaging-Noise Measurements. 2013. http://www.iso.org/iso/ home/store/catalogue_ics/ catalogue_detail_ics.htm?csnumber=59420 (10 March 2019).

ISO/CIE 11664-6 (2014). Colourimetry - Part 6: CIEDE2000 ColourDifference Formula.

Jin, E.W., Phillips, J.B., Farnand, S., Belska, M., Tran, V., Chang, E. Wang, Y., Tseng, B., 2017, Towards the development of the IEEE P1858 CPIQ standard-A validation study. Electronic Imaging, 2017(12), 88-94. doi.org/10.2352/ISSN.2470-1173.2017.12.IQSP-249.

Kolev, K., Tanskanen, P., Speciale, P., Pollefeys, M., 2014. Turning mobile phones into 3D scanners. 2014 IEEE CVPR Proceedings, 3946 3953. doi.org/10.1109/CVPR.2014.504

Kos, A., Tomažič, S., Umek, A., 2016. Evaluation of Smartphone Inertial Sensor Performance for Cross-Platform Mobile Applications. Sensors. 16(4), 477. doi.org/10.3390/s16040477.

Lebrun, M., 2012. An Analysis and Implementation of the BM3D Image Denoising Method. Image Processing On Line, 2, 175-213. doi.org/10.5201/ipol.2012.1-bm3d.

Lukac, R., Plataniotis, K.N., 2007. Single-Sensor Camera Image Processing, Color Image Processing -Methods and Applications; CRC: Boca Raton, FL, USA, pp. 363-392.

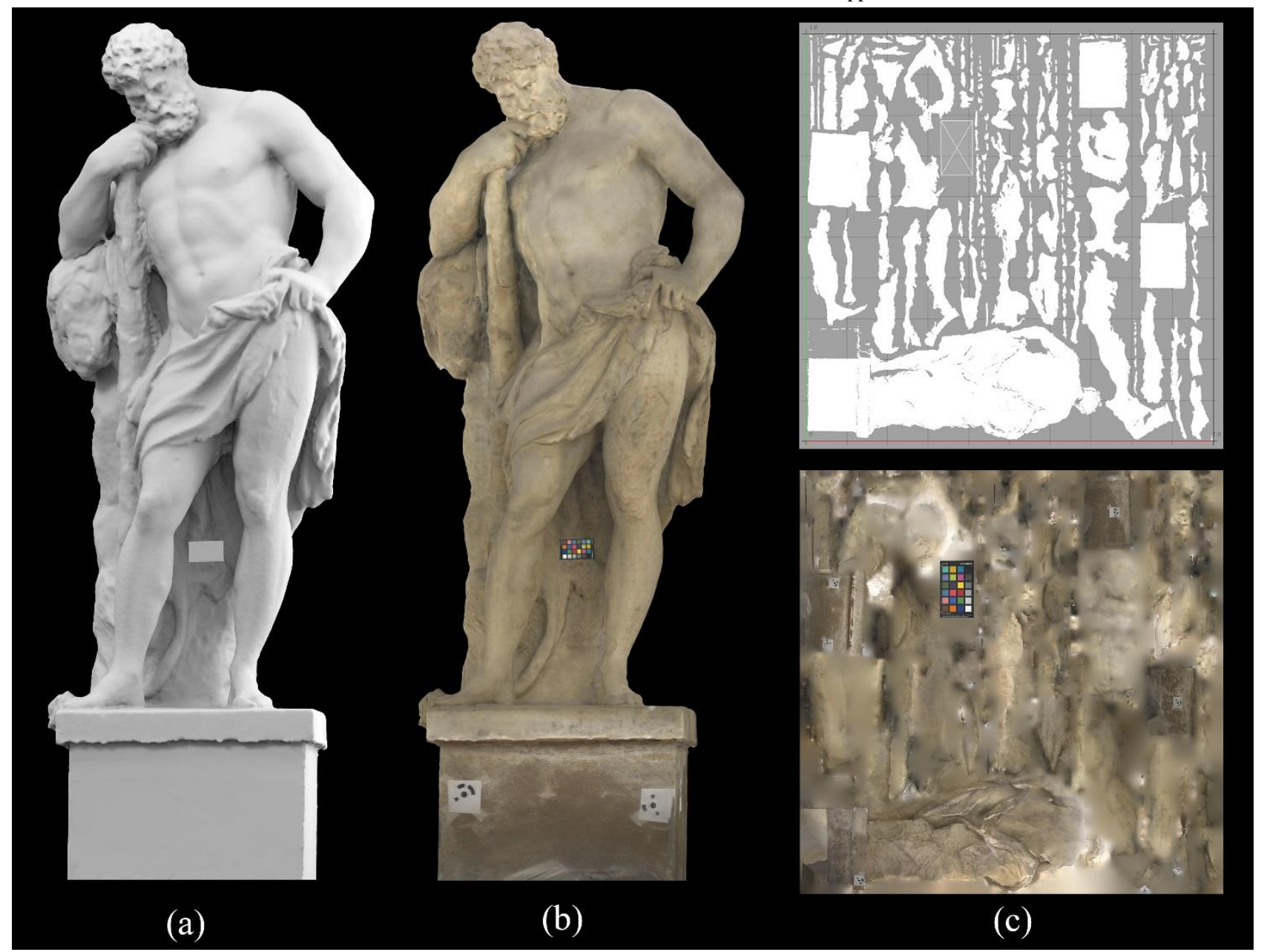

Figure 9. iPhoneX based modelling: texture map including ColorChecker Passport for color calibration 
Mokrzycki, W.S., Tatol, M., 2011. Colour difference Delta E - A survey. Machine graphics and vision, 20(4), 383-412.

Muratov, O., Slynko, Y., Chernov, V., Lyubimtseva, M., Shamsuarov A., Bucha, V., 2016. 3DCapture: 3D Reconstruction for a Smartphone, 2016 IEEE CVPRW, 893-900. doi.org/10.1109/CVPRW.2016.116.

Myers, R.D., 2010. ColorChecker Passport Technical Review, revision 3.

http://www.chromaxion.com/information/ColorChecker_Passport_Tech nical_Report.pdf (6 March 2019).

Nocerino, E., Lago, F., Morabito, D., Remondino, F., Porzi, L., Poiesi, F., Rota Bulo, S., Chippendale, P., Locher, A., Havlena, M., Van Gool, L., Eder, M., Fötschl, A., Hilsmann, A., Kausch, L., Eisert, P., 2017. A smartphone-based 3D pipeline for the creative industry - The REPLICATE EU Project. Int. Arch. Photogramm. Remote Sens. Spatial Inf. Sci., XLII-2/W3, 535-541. doi.org/10.5194/isprs-archives-XLII-2W3-535-2017.

Ondruska, P., Kohli, P., Izadi, S., 2015. MobileFusion: Real-time Volumetric Surface Reconstruction and Dense Tracking On Mobile Phones. IEEE Transactions on Visualization and Computer Graphics, 21(11), 1251-1258. doi.org/10.1109/TVCG.2015.2459902.

Ottani Cavina, A. (ed), 1988. Palazzo Poggi da dimora aristocratica a sede dell'Università di Bologna. Nuova Alfa, Bologna.

Pintus, R., Pal, K., Yang, Y., Weyrich, T., Gobbetti, E., Rushmeier, H., 2016. A Survey of Geometric Analysis in Cultural Heritage. Computer Graphics, 35(1), 4-31. doi.org/10.1111/cgf.12668.

Poiesi, F., Locher, A., Chippendale, P., Nocerino, E., Remondino, F., Van Gool, L. 2017. Cloud-based collaborative 3D reconstruction using $\begin{array}{llll}\text { smartphones. } & \text { CVMP } 2017 \quad \text { Proceedings, } & 1 .\end{array}$ doi.org/10.1145/3150165.3150166.

Peltoketo, V-T., 2015. Signal to Noise Ratio and Visual Noise of Mobile Phone Cameras. Journal of Imaging Science and Technology, 59(1) 10401-1-10401-7(7)

doi.org/10.2352/J.ImagingSci.Technol.2015.59.1. 010401.

RAGS, 2018. http://www.rags-int-inc.com/phototechstuff/ colorcalibration/ (6 March 2019)

Reinhard, E., Khan, E.A., Akyuz, A.O., Johnson, G., 2008. Colour Imaging: Fundamentals and Applications. CRC Press, Boca Raton, FL, USA.

Relio $^{2}$, 2019. www.relio.it (6 March 2019).

Remondino, F., Gaiani, M., Apollonio, F., Ballabeni, A., Ballabeni, M., Morabito, D., 2016. 3D Documentation of 40 Kilometers of Historical Porticoes - the Challenge. Int. Arch. Photogramm. Remote Sens. Spatial Inf. Sci., XLI-B5, 711-718. doi.org/10.5194/isprs-archives-XLI-B5-7112016.

Sharma, G., Wu, W., Dalal, E.N., 2005. The CIEDE2000 Colordifference formula: implementation notes, supplementary test data and mathematical observations. Color Research and Application, Vol. 30(1), pp. 21-30.

Stamatopoulos C., Fraser, C. S., Cronk, S., 2012. Accuracy aspects of utilizing raw imagery in photogrammetric measurement, Int. Arch Photogramm. Remote Sens. Spatial Inf. Sci., XXXIX-B5, 387-392. doi.org/10.5194/isprsarchives-XXXIX-B5-387-2012.

Süsstrunk S., Buckley, R., Swen, S., 1999. Standard RGB color spaces. IS\&T/SID CIC Proceedings, 7, 127-134. http://infoscience.epfl.ch/ record/34089.

TRNIO, 2019. www.trnio.com (10 March 2019). 